\title{
Aplicação de ferramentas gerenciais na prática de enfermeiros da atenção básica em saúde
}

\author{
Application of management tools in the practice of nurses of basic health care
}

\begin{abstract}
Aplicación de herramientas de gestión en la práctica de enfermeras de atención básica de salud
\end{abstract}

Liwcy Keller de Oliveira Lopes Lima ${ }^{1 *}$, Braz Jacinto da Silva Junior ${ }^{1}$, Gabriela Feitosa de Souza ${ }^{1}$, Graziela da Silva Costa ${ }^{1}$.

\section{RESUMO}

Objetivo: Analisar o uso das ferramentas gerenciais de enfermagem na prática dos enfermeiros que desempenham suas atividades nas Unidades Básicas de Saúde (UBS) em um município do Pará. Métodos: Este estudo é do tipo descritivo transversal de natureza quantitativa. A população foi composta por 9 enfermeiros que atuavam nas Unidades Básicas de Saúde (UBS) daquela cidade. A coleta dos dados ocorreu através da Plataforma Digital Google forms, em formulário em escala Likert. O estudo foi aprovado pelo Comitê de Ética em Pesquisa. Resultados: Duas dimensões alcançaram médias superiores a 75 a saber "trabalho em equipe" (85) e "utilização/Conhecimento das ferramentas gerenciais no trabalho na UBS" (77), sendo consideradas como atitudes positivas pelos entrevistados. Conclusão: No presente estudo, observou-se que a aplicação das ferramentas gerenciais faz parte das atividades diárias dos enfermeiros, tendo esses profissionais uma percepção positiva quanto a temática. No entanto, acredita-se que discussões mais aprodundadas devem ser realizadas com vistas a subsidiar propostas que contribuam efetivamente para o processo de trabalho gerencial do enfermeiro.

Palavras-chave: Gestão em saúde, Enfermagem, Gerenciamento da prática profissional, Unidade Básica de Saúde.

\section{ABSTRACT}

Objective: To analyze the use of managerial nursing tools in the practice of nurses who perform their activities in Basic Health Units (UBS) in a municipality in Pará. Methods: This is a cross-sectional study of a quantitative nature. The population was composed of 9 nurses who worked in the Basic Health Units (BHU) of that city. Data collection took place through the Google Forms Digital Platform, using a Likert scale form. The study was approved by the Research Ethics Committee. Results: Two dimensions reached averages greater than 75, namely "team work" (85) and "use / knowledge of managerial tools at work in the UBS" (77), being considered as positive attitudes by the interviewees. Conclusion: In the present study, it was observed that the application of management tools is part of nurses' daily activities, with these professionals having a positive perception regarding the theme. However, it is believed that more in-depth discussions should be held in order to support proposals that effectively contribute to the managerial work process of nurses.

Keywords: Health management, Nursing, Professional practice management, Basic Health Unit.

${ }^{1}$ Faculdade de Ensino Superior da Amazônia Reunida, Redenção - PA. *E-mail: liwcykeller@yahoo.com.br 


\section{RESUMEN}

Objetivo: Analizar el uso de herramientas gerenciales de enfermería en la práctica de enfermeros que desarrollan su actividad en Unidades Básicas de Salud (UBS) de un municipio de Pará Métodos: Estudio transversal de naturaleza cuantitativa. La población estuvo compuesta por 9 enfermeras que laboraban en las Unidades Básicas de Salud (UBS) de esa ciudad. La recolección de datos se realizó a través de la Plataforma Digital Google Forms, utilizando un formulario de escala Likert. El estudio fue aprobado por el Comité de Ética en Investigación. Resultados: Dos dimensiones alcanzaron promedios superiores a 75, a saber, "trabajo en equipo" (85) y "uso / conocimiento de herramientas gerenciales en el trabajo en las SBU" (77), siendo consideradas como actitudes positivas por los entrevistados. Conclusión: En el presente estudio se observó que la aplicación de herramientas de gestión forma parte de la actividad diaria de los enfermeros, teniendo estos profesionales una percepción positiva sobre el tema. Sin embargo, se cree que se deben realizar discusiones más profundas para apoyar propuestas que contribuyan efectivamente al proceso de trabajo gerencial de las enfermeras.

Palabras clave: Gestión de la salud, Enfermería, Gestión de la práctica profesional, Unidad Básica de Salud.

\section{INTRODUÇÃO}

A expressão gerenciamento é empregada para definir as ações de direção de uma organização ou de grupo de pessoas. Tais ações sempre fizeram parte do processo de trabalho da enfermagem que ao longo dos anos, têm buscado elementos para adequar modelos administrativos ao seu cotidiano e conciliá-los com seu papel principal: o cuidado com o paciente (MADUREIRA GC, et al., 2017).

No Brasil, ainda em 1921 as funções de gerência no trabalho do enfermeiro já eram estudadas com interesse em garantir e assegurar a qualidade dos serviços prestados (NASCIMENTO SM, 2013). Neste contexto as Diretrizes Curriculares Nacionais (DCNs) para a formação em enfermagem reafirmam a preocupação de que enfermeiros sejam aptos a gerir o trabalho em saúde, sendo a liderança, a tomada de decisão, a comunicação, a educação permanente, a administração e gerenciamento apresentados entre as competências mínimas para a formação desses profissionais (BRASIL, 2001).

O trabalho do enfermeiro, em essência, remonta-se à elaboração contínua de estratégias voltadas ao gerenciamento de recursos humanos, materiais, físicos e financeiros, além da própria assistência, com objetivo primário de se obter o melhor cuidado de enfermagem consumido pelo paciente (LUZ MAP, et al., 2017). Vale mencionar que na enfermagem a ação do gerenciamento é conferida privativamente ao enfermeiro (BRASIL, 1986), que deve utilizar ferramentas como: o planejamento, o dimensionamento de pessoal o recrutamento e seleção de pessoas, a educação continuada e/ou permanente, a supervisão, avaliação de desempenho, recursos e materiais, equipamentos e instalações, além dos diferentes saberes administrativos (PEDUZZI M, et al., 2015).

Assim, as ferramentas gerenciais são essenciais, pois contribuem para a organização e avaliação dos serviços, e em resultado obtem-se melhoria da qualidade da atenção à saúde ofertada em todos os níveis assistenciais. A avaliação de serviços de saúde é necessária como elemento do cotidiano de trabalho em saúde, de modo a permitir, a identificação de fragilidades e a visualização de oportunidades de melhoria (OLIVEIRA AS, 2017; BÁO ACP, et al., 2019).

A gestão da qualidade é imprescindível para o funcionamento dos serviços de saúde, principalmente aos pertencentes a Atenção Primária em Saúde (APS), uma vez que devido ao advento da Estratégia de Saúde da Família, como modelo de atenção básica e centro ordenador da rede de atenção à saúde do Sistema Único de Saúde (SUS) fez-se necessário um modelo de gerencia que priorizasse um planejamento adequado e um fluxo de atendimento sem grandes obstáculos para o usuário (FERNANDES MC, et al., 2010; BRASIL, 2017). 
A ação gerencial em uma Unidade Básica de Saúde (UBS) caracteriza-se em grande parte pela análise do processo de trabalho, com identificação de problemas e busca de soluções para reorganização das práticas de saúde, na tentativa de alcançar as metas descritas no planejamento (FERNANDES MC, et al., 2010). Assim, nesses contextos, o gerenciamento constitui-se importante instrumento para o enfermeiro, em que o profissional deverá entender que a gerência não se baseia apenas em capacidades técnicas ou algo dedutivo, mas cientificamente por meio das ferramentas do gerenciamento (FERNANDES JC e CORDEIRO BC, 2018).

Em estudo realizado nas UBS do interior do Paraná, observou-se que as ferramentas mais referidas pelos enfermeiros foram para direcionamento e controle e avaliação do serviço, $32,0 \%$ e $17,0 \%$, respectivamente. Entretanto, os resultados apontaram para uma fragilidade no tocante à prática gerencial desses profissionais, que encontram-se sobrecarregados de funções administrativas e assistenciais, além de apresentarem dificuldades em reconhecer e utilizar ferramentas que auxiliem no processo gerencial que podem estar associadas ao baixo conhecimento sobre esse tema e à sobrecarga de atividades que poderiam ser delegadas (OLIVEIRA SA, et al., 2017).

Os enfermeiros apresentam inúmeras dificuldades para exercício da gerência, o que torna importante identificar os obstáculos que atrapalham o trabalho do profissional, uma vez que uma boa atuação na gerência pode trazer melhorias na assistência à saúde e na produção da equipe, principalmente quando se refere a APS (MADUREIRA GC, et al., 2017).

Assim, acredita-se que o conhecimento sobre as ferramentas gerenciais utilizadas pelo enfermeiro pode tornar sua prática mais efetiva, principalmente na atenção primária. Diariamente, desafios são apresentados ao mundo corporativo, e cabe ao enfermeiro, dentro da realidade atual, buscar alternativas que respondam às necessidades dos recursos humanos de enfermagem na organização de saúde.

Diante disso, o presente estudo objetivou analisar o uso das ferramentas gerenciais de enfermagem na prática dos enfermeiros que desempenham suas atividades nas UBS em um município do Pará, verificando o conhecimento dos enfermeiros sobre essas ferramentas e as dificuldades encontradas por esses para sua utilização na prática.

\section{MÉTODOS}

Este estudo é do tipo descritivo transversal de natureza quantitativa. A população foi composta por enfermeiros que atuavam nas 12 Unidades Básicas de Saúde (UBS) que compõem a atenção básica em um município do Pará. Foram incluídos no estudo todos os enfermeiros que exerciam atividades gerenciais e assistenciais nas unidades de saúde e que consentiram em participar da pesquisa por meio da concordância do Termo de Consentimento Livre e Esclarecido e resposta ao questionário enviado por dispositivo eletrônico. Os enfermeiros que no período de coleta de dados encontravam-se afastados de suas atividades laborais (licença, férias, entre outros) foram excluídos do estudo.

Os dados foram coletados por meio de instrumento estruturado autoaplicável, através da Plataforma Digital Google forms, onde foi considerado duas seções divididas entre o perfil dos entrevistados e as dimensões das ferramentas gerenciais além de duas questões discursivas em que o profissional deveria descrever sua concepção sobre ferramenta gerencial e as dificuldades encontradas em sua prática para a implantação dessas.

A sessão referente as dimensões das ferramentas gerenciais composta por 32 assertivas sendo distribuídas em: conhecimento sobre gerenciamento, trabalho desempenhado na UBS, a infraestrutura da UBS, trabalho em equipe e a utilização/conhecimento das ferramentas gerenciais no trabalho na UBS em que os participantes indicaram sua perspectiva em relação às mesmas em escala Likert (discordo totalmente, discordo, não tenho opinião, concordo, concordo totalmente). A escala Likert é uma escala psicométrica que faz medições sobre o grau de conformidade de uma pessoa em relação a uma pergunta. Sendo que, quanto maior for o escore da escala, mais positiva é a atitude do profissional frente a pergunta, com exceção dos itens que possuiam escore reverso. 
Para analisar as respostas, foram efetuados dois cálculos. O primeiro cálculo foi para converter a escala de Likert e o segundo, para determinar a média das dimensões. Assim, no primeiro cálculo, os escores de cada item da escala de Likert foram convertidos para uma escala de 100 pontos, na qual um escore de 100 é considerado o desejável, assumindo, portanto, a seguinte pontuação de forma crescente: discordo totalmente $=0$; discordo $=25$; não tenho opinião $=50$; concordo $=75$; concordo totalmente $=100$. Com estes valores, as respostas de cada dimensão foram somadas e divididas pelo número de questões, resultando em uma variação de 0 a 100, sendo considerada uma atitude positiva quando este resultado foi igual ou maior a $75 \mathrm{em} \mathrm{100.} \mathrm{Já} \mathrm{no} \mathrm{segundo} \mathrm{cálculo,} \mathrm{foram} \mathrm{somados} \mathrm{os} \mathrm{valores} \mathrm{de} \mathrm{cada} \mathrm{item} \mathrm{de} \mathrm{resposta,}$ incluindo o escore reverso calculado e, por média simples, obteve-se a média de cada dimensão.

Quanto às perguntas abertas, foram reorganizadas a partir das respostas dos entrevistados que atenderam efetivamente as questões apresentadas, suprimindo as palavras que fogem da pergunta. Essas respostas foram apresentadas permeio da similaridade no formato de tabelas.

O estudo foi avaliado e aprovado pelo Comitê de Ética em Pesquisa (CEP) da Faculdade de Ensino Superior da Amazônia Reunida - FESAR, com número de parecer 4.126.564 e CAAE: 32166720.7.0000.8104.

\section{RESULTADOS E DISCUSSÃO}

Do total de doze enfermeiros que compõem o quadro da saúde básica do municipio de Redenção no estado do Pará, nove $(75,0 \%)$ aceitaram participar e responderam aos questionários desta pesquisa. 0 perfil dos enfermeiros entrevistados estão apresentados a seguir (Tabela 1).

Tabela 1 - Perfil dos enfermeiros entrevistados sobre os aspectos relacionados ao uso de ferramentas gerenciais na prática dos enfermeiros na atenção básica em saúde (UBS), $n=9$.

\begin{tabular}{|c|c|c|}
\hline Variáveis & $\mathbf{N}$ & $\%$ \\
\hline \multicolumn{3}{|l|}{ Sexo } \\
\hline Feminino & 8 & 88,9 \\
\hline Masculino & 1 & 11,1 \\
\hline \multicolumn{3}{|l|}{ Estado civil } \\
\hline Solteiros & 2 & 22,2 \\
\hline Divorciado & 1 & 11,1 \\
\hline Casados & 5 & 55,6 \\
\hline Outros & 1 & 11,1 \\
\hline \multicolumn{3}{|l|}{ Faixa etária } \\
\hline 20 a 30 anos & 2 & 22,2 \\
\hline 30 a 40 anos & 6 & 66,7 \\
\hline 40 a 50 anos & 1 & 11,1 \\
\hline Acima de 50 anos & 0 & 0 \\
\hline \multicolumn{3}{|l|}{ Tempo de atuação } \\
\hline Menos que 1 ano & 2 & 22,2 \\
\hline 1 a 5 anos & 4 & 44,4 \\
\hline Entre 05 e 10 anos & 2 & 22,2 \\
\hline Mais de 10 anos & 1 & 11,1 \\
\hline \multicolumn{3}{|l|}{ Formação complementar* } \\
\hline Cursos de curta duração (até $60 \mathrm{~h}$ ) & 4 & 26,6 \\
\hline Capacitação/aperfeiçoamento (mínimo 100h) & 3 & 20 \\
\hline Esp. na área de gestão/gerenciamento/ administração & 0 & 0 \\
\hline Esp. na área de saúde pública ou afins & 2 & 13,3 \\
\hline Especialização em outras áreas & 4 & 26,6 \\
\hline Mestrado & 0 & 0 \\
\hline Doutorado & 0 & 0 \\
\hline Pós- doutorado & 0 & 0 \\
\hline Outros & 2 & 13,3 \\
\hline Total & 9 & 100 \\
\hline
\end{tabular}


O enfermeiro é definido como "um gerenciador de cuidados" com base nas suas atividades profissionais que passa pela esfera gerencial e assistencial bem como, nas atividades de ensino e pesquisa (TREVISO P, et al., 2017). Neste sentido Soares Ml, et al. (2016) afirmam que na enfermagem, os saberes gerenciais do enfermeiro contempla várias formas de abordar as atividades inseridas no seu processo de aprendizado no decorrer de sua formação.

Observa-se que a maioria dos entrevistados são do sexo feminino semelhante ao descrito na literatura em que a predominância feminina na enfermagem encontra-se entre 86,2\%. Esta predominância é compartilhada por outros autores, reproduzindo a característica histórica da enfermagem, profissão exercida quase que exclusivamente por mulheres desde os seus primórdios (MARTINS C, 2006; SILVA JDF, 2018).

O maior percentual da faixa etária apresentada nesta pesquisa é de 30 a 40 anos de idade $(44,4 \%)$, percentual semelhante foram encontrados nas pesquisas realizada com os enfermeiros que atuam na atenção primária à saúde de um município do sul do Brasil na região de tríplice fronteira, 50\% e 61,9\% (LUZ MAP, et al., 2017; OLIVEIRA SA, et al., 2017). Outro dado levantado foi a situação civil dos entrevistados, sendo que $55,6 \%(n=05)$ declararam serem casados, índices semelhantes foram apresentados na pesquisa de Oliveira JF, et al. (2019), sendo 59\% casados ou viviam com alguém.

Quanto aos dados da formação complementar dos entrevistados, seis (66,6\%) afirmaram possuir especialização na área de saúde pública ou outras áreas, sendo a titulação mais mencionada. Entretanto, nenhum dos entrevistados relatou possuir curso específico na área de gestão, gerenciamento ou administração. Fato de extrema relevância, se levarmos em conta as responsabilidades do cargo, pois demonstra que a instituição não considerou este critério para a escolha do profissional para o cargo. Contudo, os números também mostraram pouco investimento dos profissionais para o seu próprio aprimoramento na área gerencial (SILVA VLDS, et al., 2017).

Entre as cinco dimensões avaliadas pelo instrumento, a atitude com média mais elevada foi "trabalho em equipe" $(85,42)$, enquanto as dimensões que apresentaram as menores médias de escores foram "trabalho desempenhado na UBS" $(68,65)$ e "conhecimento sobre gerenciamento" $(63,89)$, representando as atitudes menos valorizadas pelos profissionais (Figura 1).

Figura 1 - Média de escores das dimensões obtidos a partir da avaliação do uso de ferramentas gerenciais na prática dos enfermeiros nas Unidades Básicas em Saúde (UBS), n=9.

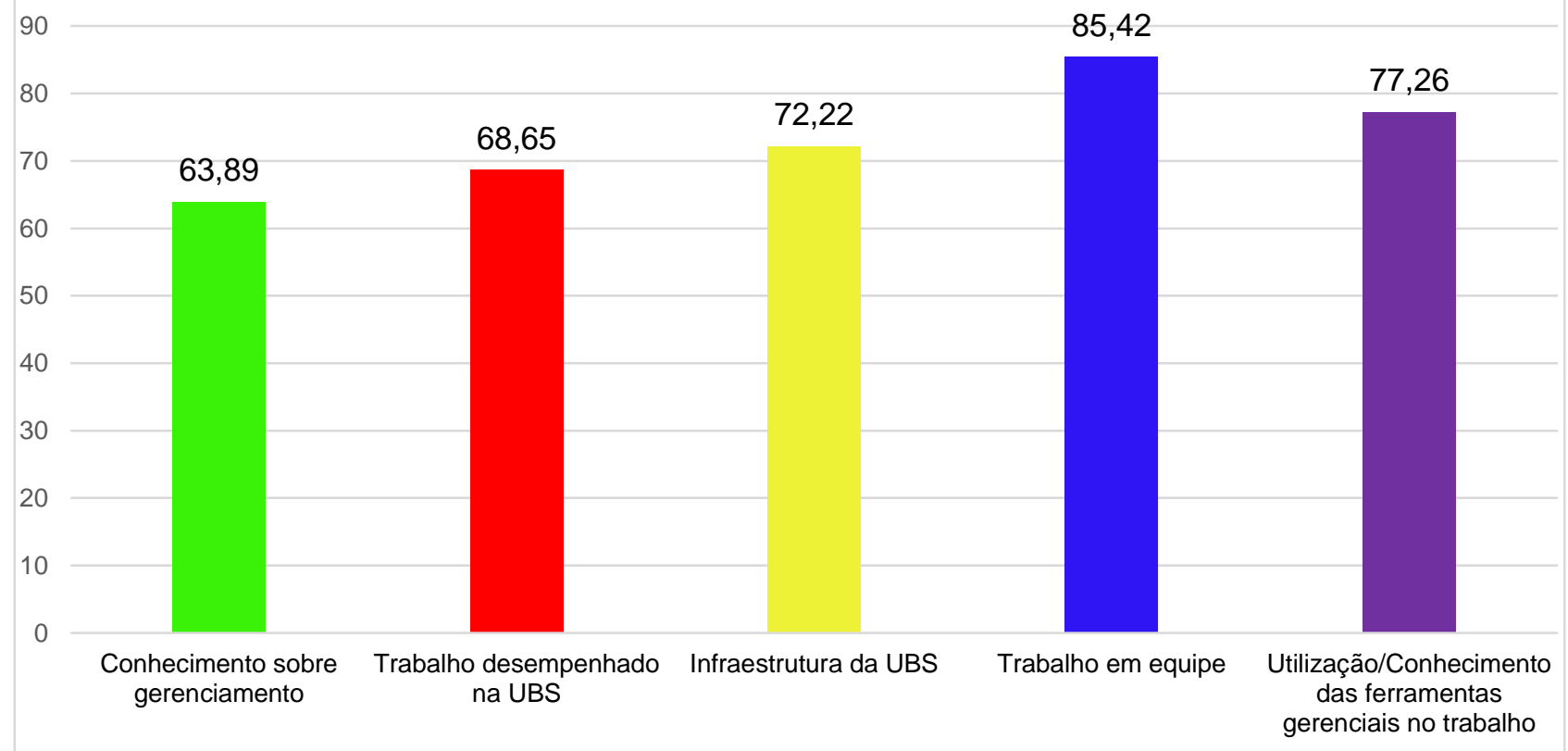

Fonte: Lima LKOL, et al., 2020. 
A dimensão "trabalho em equipe" $(85,42)$, foi considerada atitude positiva ultrapassando a média de 75 necessárias para ser considerada como tal. Nesta dimensão foi verificado que 0 item com escore mais alto $(94,44)$ estava relacionado com a seguinte afirmação: eu faço ações voltadas para o treinamento da equipe e o item com escore mais baixo $(77,78)$, relacionava-se a afirmação: Sinto-me encorajado(a) pelos meus colegas para tomada de decisão.

Dessa forma, fica demonstrado a importância das ferramentas de gestão nas atividades dos enfermeiros contribuindo diretamente para a organização e avaliação dos serviços prestados nas UBS. É de fundamental importância a preparação dos profissionais que atuam na área da saúde para que respondam efetivamente às demandas encontradas ao longo do exercício profissional (OLIVEIRA SA, et al., 2017; DIAS AKG, 2017).

O enfermeiro tem papel de destaque dentre os profissionais que compõem a equipe de enfermagem, Sendo, cada dia mais cobrado desses profissionais a habilidade de trabalhar em equipe considerando as necessidades da mesma, bem como de seus clientes, familiares e da própria instituição. Baseado nesta afirmação, espera-se dos enfermeiros maior capacitação para lidar com os desafios e conflitos da profissão, bem como, o gerenciamento dos ambientes de trabalho (TORRES E, 2011; SOARES MI, et al., 2016).

Nos resultados encontrados entre as dimensões a dimensão "conhecimento sobre gerenciamento" $(63,89)$ apresentou a menor média, essa foi a dimensão considerada como a menor atitude positiva pelos entfermeiros. O item referente a afirmativa: durante a graduação cursei disciplinas voltadas para administração/gerenciamento apresentou maior escore (75) e a afirmativa: obtive conhecimentos sobre as ferramentas gerenciais apenas após minha formação se destacou de forma negativa dentro dessa dimensão, com escore reverso de 50 .

Esse resultado abaixo da média (75) evidenciando assim, a fragilidade no tocante à prática gerencial dos enfermeiros na atenção básica, e que esses se encontram sobrecarregados de funções administrativas e assistenciais. Quando se verifica a proposta das Diretrizes Curriculares para o curso de enfermagem, o seu objetivo é dotar os profissionais de conhecimentos requeridos para o exercício das seguintes competências e habilidades gerais: atenção à saúde, tomada de decisões, comunicação, liderança, administração e gerenciamento e educação permanente (BRASIL, 2001; OLIVEIRA AS, et al., 2017).

$\mathrm{Na}$ dimensão "utilização/conhecimento das ferramentas gerenciais no trabalho na UBS" $(77,26)$ semelhante a dimensão "trabalho em equipe" foi considerada atitude positiva pelos entrevistados, o que demonstra a importância da aplicabilidade da formação em enfermagem. A afirmativa: A utilização das ferramentas gerenciais contribuem para a organização dos serviços de saúde. Apresentou a maior média nesta dimensão $(97,22)$ e a afirmativa: Durante a minha atuação na unidade básica de saúde utilizo o recrutamento e seleção de pessoal. Apresentou a menor média desta dimensão $(47,22)$.

A dimensão 5 "Utilização/conhecimento das ferramentas gerenciais" também foi apresentada em quadro separado por apresentar maior número de questões, bem como, as questões reversas (Quadro 1). 
Quadro 1 - Escore médio da percepção dos enfermeiros quanto à dimensão 5 "utilização/conhecimento das ferramentas gerenciais no trabalho na UBS".

\begin{tabular}{|c|c|c|c|c|c|c|c|}
\hline \multicolumn{2}{|r|}{ QUESTÕES } & \multirow{2}{*}{$\begin{array}{c}\text { DT } \\
\mathbf{0} \\
-\end{array}$} & \multirow{2}{*}{$\begin{array}{c}\text { D } \\
25 \\
25\end{array}$} & \multirow{2}{*}{$\begin{array}{c}\text { NTO } \\
50 \\
-\end{array}$} & \multirow{2}{*}{$\begin{array}{c}C \\
75 \\
150\end{array}$} & \multirow{2}{*}{$\begin{array}{c}\text { CT } \\
100 \\
600\end{array}$} & \multirow{2}{*}{$\begin{array}{c}\begin{array}{c}\text { Média } \\
\text { Simples }\end{array} \\
86,11\end{array}$} \\
\hline A17 & No meu cotidiano utilizo ferramentas gerenciais para execução das minhas atividades. & & & & & & \\
\hline A18 & Durante a minha atuação na unidade básica de saúde utilizo o planejamento. & - & - & - & 150 & 700 & 94,44 \\
\hline A19 & Durante a minha atuação na unidade básica de saúde utilizo o dimensionamento de pessoal. & - & 25 & - & 300 & 400 & 80,55 \\
\hline A20 & $\begin{array}{l}\text { Durante a minha atuação na unidade básica de saúde utilizo o recrutamento e seleção de } \\
\text { pessoal. }\end{array}$ & 0 & 75 & 50 & 300 & - & 47,22 \\
\hline A21 & Durante a minha atuação na unidade básica de saúde utilizo a educação continuada/permanente. & - & - & - & 225 & 600 & 91,66 \\
\hline A22 & Durante a minha atuação na unidade básica de saúde utilizo a supervisão. & - & - & - & 150 & 700 & 94,44 \\
\hline A23 & Durante a minha atuação na unidade básica de saúde utilizo avaliação de desempenho. & - & - & - & 450 & 300 & 83,33 \\
\hline A24 & A utilização das ferramentas gerenciais contribui para direcionamento dos serviços de saúde. & - & - & - & 225 & 600 & 91,66 \\
\hline A25 & A utilização das ferramentas gerenciais contribui para a organização dos serviços de saúde. & - & - & - & 75 & 800 & 97,22 \\
\hline A26 & A utilização das ferramentas gerenciais contribui na melhora do planejamento da assistência. & - & - & - & 150 & 700 & 94,44 \\
\hline A27 & Quando utilizo as ferramentas gerenciais percebo melhora na qualidade do atendimento. & - & - & - & 150 & 700 & 94,44 \\
\hline A28 & $\begin{array}{l}\text { A utilização das ferramentas gerenciais na organização é importante para estabelecer a estrutura } \\
\text { e executar os planos, determinando o mais adequado a ser oferecido ao paciente e agrupando as } \\
\text { atividades para atingir as metas da unidade básica. }\end{array}$ & - & - & - & 225 & 600 & 91,66 \\
\hline A29 & $\begin{array}{l}\text { A qualidade no trabalho gerencial dependerá do meu conhecimento sobre funções e instrumentos } \\
\text { gerenciais, e da forma como utilizo esses recursos no dia a dia. }\end{array}$ & - & - & - & 300 & 500 & 88,88 \\
\hline A30 & $\begin{array}{l}\text { No uso das ferramentas gerenciais tenho dificuldade quando se trata de conduzir e gerenciar a } \\
\text { equipe. }\end{array}$ & 0 & 125 & 50 & 75 & - & $27,77^{*}$ \\
\hline A31 & $\begin{array}{l}\text { No uso das ferramentas gerenciais tenho dificuldade quando se trata de gerenciamento de } \\
\text { recursos e materiais. }\end{array}$ & - & 125 & 150 & 75 & - & $38,88^{*}$ \\
\hline A32 & $\begin{array}{l}\text { Executando as atividades na UBS tenho dificuldade quanto ao uso das ferramentas gerenciais } \\
\text { devido ao pouco conhecimento das mesmas. }\end{array}$ & 0 & 100 & 50 & 225 & - & $41,66^{\star}$ \\
\hline
\end{tabular}

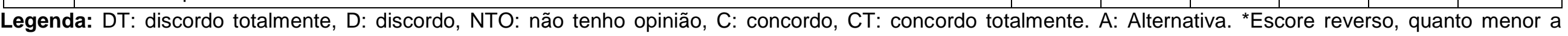
média, mais a atitude será considerada positiva.

Fonte: Lima LKOL, et al., 2020. 
O resultado acima descrito apresenta a efetiva utilização dos conhecimentos das ferramentas gerenciais por parte da grande maioria dos enfermeiros. Em pesquisa sobre as ferramentas gerenciais na prática de enfermeiros da atenção básica em saúde, destacam que apenas $48 \%$ de seus entrevistados afirmaram utilizar esse conhecimento em suas atividades profissionais. Existe uma necessidade da utilização dos conhecimentos adquiridos durante a formação em enfermagem no sentido de cumprir com a missão social e humana, desse profissional em promover e proteg er a saúde da população (FERNANDES, et al., 2010; OLIVEIRA AS, et al., 2017).

Treviso $\mathrm{P}$, et al. (2017) asseguram que a graduação, sem dúvida, é uma grande aliada no desenvolvimento do conhecimento e em sua aplicação na vida profissional do enfermeiro. Pois é na graduação que os futuros enfermeiros precisam adquirir tais conhecimentos extremamente necessários em sua atuação profissional. A falta de conhecimento dessas ferramentas por parte dos enfermeiros em sua graduação corrobora para as dificuldades apresentadas nas respostas dos entrevistados.

As diretrizes curriculares nacionais do curso de graduação em enfermagem enfatizam que a graduação deve conferir terminalidade e capacidade acadêmica e/ou profissional, considerando as demandas e necessidades prevalentes e prioritárias da população conforme o quadro epidemiológico do país/região. Uma preocupação que precisa ser superada ainda na graduação, ou seja, a eliminação da dicotomia existente entre o cuidado assistencial e o gerenciamento, já que a administração faz parte do processo de trabalho do enfermeiro. Essa dicotomia se apresenta em toda vida profissional dos enfermeiros e os mesmos precisaram atender as exigências do mercado de trabalho (BRASIL, 2001; TREVISO P, et al., 2017).

A dimensão: "trabalho desempenhado na UBS" $(68,65)$, destacou a afirmativa: gosto do meu trabalho que alcançou a média de $100 \%$. A satisfação na realização das atividades profissionais é um dos requisitos de extrema importância, principalmente nas atividades que lidam diretamente com os seres humanos. Resultados semelhantes foram encontrados na pesquisa realizada por Moreira JM, et al. (2019) a realização profissional, gratificação pessoal por suas atividades, orgulho pelo que faz, satisfação e identificação com suas tarefas, como sendo vivências que causam prazer nos profissionais foram os temas mais citados.

Acrescentam-se a esses fatores promovedores de satisfação no ambiente de trabalho dos profissionais de enfermagem os apresentados por Ozanam MAQ, et al. (2019) a saber: o bom relacionamento interpessoal, reconhecimento e valorização do trabalho, prazer no que faz e autonomia nas tomadas de decisões no processo de enfermagem. Demonstrando assim, a necessidade da utilização das ferramentas gerenciais em suas atividades profissionais.

Ainda nesta dimensão, duas assertivas apresentaram escore médio baixo, a saber: eu sou pouco efetivo ao trabalho quando estou cansado e eu me sentiria seguro sendo tratado nesta unidade como paciente apresentaram $(52,78)$ respectivamente. A questão do cansaço ou esgotamento profissional que sofrem os enfermeiros pode ser atenuada com o reconhecimento por parte dos usuários dos serviços básicos de saúde (MOREIRA JM, et al., 2019).

Com relação a dimensão "infraestrutura da UBS" $(72,22)$ que alcançou a terceira melhor média entre as dimensões estudadas, a afirmação: a infraestrutura da UBS favorece para desenvolvimento das práticas em saúde ganhou destaque, apresentando escore médio elevado $(80,56)$ o que é confirmado por Leite DF, et al. (2014) a infraestrutura é um dos aspectos fundamentais para o desenvolvimento do trabalho. Wiśniewski D, et al. (2015) chama a atenção ainda para a promoção de melhores condições laborais, seja no âmbito da estrutura do local de trabalho como da relação entre os membros da equipe.

Apoio esse que fica claro com a afirmativa: equipamentos e a instalação física estão presentes na instituição e são constantemente melhoradas, com total apoio da administração superior, alcançou o escore médio de 63,89. Esse índice é significativo uma vez que os entrevistados atribuem atenção para essa questão. Oliveira JF, et al. (2019) se refere a esses fatores como pontos negativos quando não observados pelos gestores municipais sendo a falta de recursos para materiais e alimentos, precariedade na infraestrutura do serviço, dificuldade de estabelecer um sistema de referência junto às unidades básicas de saúde do município. 
Em perguntas abertas foi questionado aos enfermeiros "Quais dificuldades encontradas no seu cotidiano relacionadas a aplicação dasferramentas gerenciais?" Obteve-se a resposta de apenas oito entrevistados estando entre as dificuldades mais mencionadas a falta de estrutura e equipamentos adequados (37,5\%) para a implantação de ferramentas gerenciais, seguida pela falta de capacitação profissional e falta de incentivo e apoio do poder público com $25 \%$ respectivamente (Tabela 2).

Tabela 2 - Descrição das dificuldades pontuadas pelos enfermeiros para a aplicação das ferramentas gerenciais no cotidiano de trabalho nas UBS $n=14$.

\begin{tabular}{lcc}
\hline DIFICULDADES DESCRITAS & $\mathbf{N}$ & $\mathbf{0}$ \\
\hline Falta de estrutura e equipamentos adequados & 3 & 37,5 \\
Falta de capacitação profissional, & 2 & 25,0 \\
Falta de incentivos e apoio do poder público & 2 & 25,0 \\
Falta de comprometimento dos funcionários & 1 & 12,5 \\
Sobrecarga de funções administrativas & 1 & 12,5 \\
Falta de comuniçção & 1 & 12,5 \\
Ativismo no trabalho & 1 & 12,5 \\
Adaptação pessoal nas mudanças & 1 & 12,5 \\
Implantação das ferramentas & 1 & 12,5 \\
Nenhuma & 1 & 12,5 \\
\hline Total & $\mathbf{1 4}$ & $\mathbf{1 0 0}$ \\
\hline
\end{tabular}

Fonte: Lima LKOL, et al., 2020.

Em relação as dificuldades descritas pelos enfermeiros para a aplicação das ferramentas gerenciais em seu cotidiano de trabalho observou-se uma diversificações nas respostas, o que confirma a observação de Madureira GC, et al. (2016) "os enfermeiros apresentam inúmeras dificuldades para exercício da gerência" essas difucldades de acordo com os entrevistados vão desde a sobrecarga de funções administrativas, a falta de comunicação, o ativismo e a falta de apoio do poder público contrastando com a simples afirmação "nenhuma".

Destacam-se às questões da responsabilidades dos centro formadores, em instrumentalizar o futuro profissional para o desenvolvimento de tais saberes e a necessidade do ensino se aproximar mais dos problemas da prática da enfermagem a necessidade de desenvolver um feedback permanente às gerências dos serviços de enfermagem, através de atividades variadas como grupos de estudos, oficinas, projeto de treinamento e capacitação para esses profissionais, e o planejamento centrado na melhoria dos processos de enfermagem (TORRES E, et al., 2011; SOARES MI, et al., 2016; TREVISO P, et al., 2017).

Conforme, apresentado por Treviso P, et al. (2017) existe uma determinada confusão quando se trata da conceituação de competência e ferramentas utilizadas no processo de cuidado da enfermagem. Com base na afirmativa de Soares MI, et al. (2016) os objetos de trabalho gerencial do enfermeiro são a organização do trabalho e os recursos humanos de enfermagem. A concepção de ferramentas gerenciais apresentadas pelos enfermeiros entrevistados destaca os recursos e as funções administrativas desenvolvidas em suas atividades nas UBS (52,5\%), as mesmas têm servido como um arsenal de apoio à função gerencial do enfermeiro (OLIVEIRA AS, 2017).

Também foi realizado a seguinte pergunta aos entrevistados: "Em sua concepção, o que são ferramentas gerenciais? Entre os oito profissionais que responderam a essas perguntas, destacou-se as seguintes respostas (Tabela 3 ).

Tabela 3 - A concepção de ferramentas gerenciais dos enfermeiros das UBS $n=9$.

\begin{tabular}{lcc}
\hline CONCEPÇÕES SOBRE AS FERRAMENTAS & $\mathbf{N}$ & $\%$ \\
\hline São ferramentas essenciais de auxilio nas atividades dos enfermeiros & 3 & 37,5 \\
Funções administrativas, argumentar e concretizar mudanças, fazer & 3 & 37,5 \\
negociações, solucionar conflitos, criar e empreender & 2 & 25,0 \\
São recursos para organizar e agilizar o serviço do enfermeiro & 1 & 12,5 \\
São utilizadas para melhor atender aos pacientes & $\mathbf{9}$ & $\mathbf{1 0 0}$ \\
\hline Total
\end{tabular}

Legenda: ${ }^{*} \mathrm{O}$ número será maior que 8 (a quantidade de entrevistados).

Fonte: Lima LKOL, et al., 2020. 
Com relação às concepções da definição de ferramentas gerenciais destacou-se às questões referentes às funções administrativas (37,5\%) e ferramentas essenciais para organizar e agilizar o serviço (37,5\%), em geral os entrevistados visualizam as ferramentas gerenciais como "ações" e complementam que as ferramentas são recursos valiosos. Resultados semelhantes foram percebidos por Soares MI, et al. (2016) ao afirmarem que o planejamento e a organização das ações foram percebidos em nosso estudo como um saber gerencial imprescindível para o cotidiano de trabalho do enfermeiro que podem ser adquiridos durante a graduação.

O estudo realizado apresentou algumas limitações significativas com relação à sua população e amostra. A princípio, foram identificados o universo da pesquisa e enviado a solicitação para que respondessem aos questionários da pesquisa. Outra limitação importante se refere ao tamanho da amostra alcançada, o que permite considerar os resultados encontrados apenas para a população em questão.

Os estudos sobre a aplicação das ferramentas gerenciais nas atividades dos enfermeiros são fundamentais para a compreensão dos limites e dificuldades enfrentadas por estes profissionais. Novas abordagens podem ser desenvolvidas com a finalidade de compreender os fatores limitantes da aplicação dessas ferramentas. Estudos envolvendo a efetividade das ações, os obstáculos mais recorrentes, as demandas por capacitações e outros podem contribuir ainda mais com o avanço dessa temática.

\section{CONCLUSÃO}

$\mathrm{Na}$ análise geral, a aplicação das ferramentas gerenciais nas atividades dos enfermeiros mostrou-se extremamente presente no dia a dia desses profissionais. A familiarização desse tema ao longo da graduação profissional apresenta-se como fundamental no processo de capacitação. A percepção dos enfermeiros é positiva quanto à aplicação dessas ferramentas. Contudo, acredita-se que discussões mais aprodundadas devem ser realizadas com vistas a subsidiar propostas que contribuam efetivamente para 0 processo de trabalho gerencial do enfermeiro, principalmente na atenção básica. Além disso, os resultados obtidos podem contribuir tanto para a comunidade acadêmico-científica quanto para os gestores municipais e regionais, fornecendo subsídios para desenvolvimento de estratégias e ações que priorizem a qualidade do cuidado.

\section{REFERÊNCIAS}

1. BÁO ACP, et al. Indicadores de qualidade: ferramentas para o gerenciamento de boas práticas em saúde. Revista Brasileira de Enfermagem, 2019; 72(2), 360-366.

2. BRASIL, 1986. Senado Federal; Subsecretaria de informações. Lei no 7.498, de 25 de junho de 1986. Dispõe sobre a regulamentação do exercício da enfermagem.

3. BRASIL, 2001. Ministério da educação. Conselho Nacional de Educação, Câmara de Educação Superior. Resolução CNE/CES no 3 de 7 de novembro de 2001: Diretrizes Curriculares Nacionais do Curso de Graduação em Enfermagem.

4. BRASIL, 2001. Conselho Nacional de Educação. Câmara de Educação Superior. Resolução CNE/CES 3/2001. Diário Oficial da União. Seção 1, p. 37

5. BRASIL, 2013. Ministério da saúde. Conselho nacional de saúde. Resolução no 466, de 12 de dezembro de 2012.

6. BRASIL, 2017. Ministério da Saúde. Portaria no. 2.436 de 21 de setembro de 2017.

7. DIAS AKG, et al. A percepção dos enfermeiros em relação ao seu papel gerencial no âmbito hospitalar. Rev. enferm. UFPE on line, 2017; 11(5): 2185-2194.

8. FERMINO V, et al. Estratégia Saúde da Família: gerenciamento do cuidado de enfermagem. Revista Eletrônica de Enfermagem, 2017; 19(5).

9. FERNANDES JC, CORDEIRO BC. O gerenciamento de Unidades Básicas de Saúde no olhar dos enfermeiros gerentes. Revista de Enfermagem. UFPE on line, 2018; 12(1):194-202,.

10. FERNANDES MC, et al. Análise da atuação do enfermeiro na gerência de unidades básicas de saúde. Revista Brasileira de Enfermagem, 2010; 63(1): 11-15

11. FRANCO SC, et al. Análises pelos discentes de uma escola médica das atividades de integração ensino-serviço na graduação. Revista Eletrônica Acervo Saúde, 2019; (33), e921.

12. HAUSMANN M, PEDUZZI M. Articulação entre as dimensões gerencial e assistencial do processo de trabalho do enfermeiro. Texto \& Contexto-Enfermagem, 2009; 18(2):258-265,

13. LEITE DF, et al. Qualidade de vida no trabalho de profissionais do NASF no município de São Paulo. Physis Revista de Saúde Coletiva, 2014; 24(2): 507-525. 
14. LUZ MAP, et al Competências gerenciais: opinião de enfermeiros no contexto hospitalar. Revista Eletrônica Gestão \& Saúde, Brasília, 2017; 8(3): 521-538.

15. MACHADO M. Perfil da enfermagem no Brasil: relatório final. FIOCRUZ/COFEN, 2017; 28(1).

16. MADUREIRA GC, et al. Reflexão sobre a enfermagem e o gerenciamento das unidades básicas de saúde. Rev. Baiana Saúde Pública, 2017; 40(4):848-861.

17. MARTINS C, et al. Perfil do enfermeiro e necessidades de desenvolvimento de competência profissional. Texto \& Contexto-Enfermagem, 2006; 15(3):472-478.

18. MENEZES PIFB, DINNOCENZO M. Dificuldades vivenciadas pelo enfermeiro na utilização de indicadores de processos. Revista Brasileira de Enfermagem, 2013; 66(4):571-577.

19. MOREIRA JM, et al. Fatores desencadeadores de (in)satisfação no trabalho dos enfermeiros da atenção básica de saúde. Revista Ciência y Enfermeria, 2019; 25(12):0-0

20. MULLER LA, et al. Percepção de enfermeiros sobre o processo de gestão em um hospital universitário. Rev. Enferm. UFPE on line, 2013; 11(12):5321-5327.

21. NASCIMENTO SM. As funções gerenciais do enfermeiro no cotidiano da assistência hospitalar. 2013.

22. OLIVEIRA JF, et al. Satisfação profissional e sobrecarga de trabalho de enfermeiros da área de saúde mental. Ciência \& Saúde Coletiva,2019; 24(7):2593-2599.

23. OLIVEIRA SA, et al. Ferramentas gerenciais na prática de enfermeiros da atenção básica em saúde. Revista. Administração e Saúde, 2017; 17(69).

24. OZANAM MAQ, et al. Satisfação e insatisfação no trabalho dos profissionais de enfermagem. Brazilian Journal of Development, Curitiba, 2019; 5(6):6156-6178.

25. PEDUZZI M, et al. Gerenciamento em enfermagem. Guanabara Koogan, 2ª Ed. 2015.

26. ROMAGNOLLI APB, et al. Gestão de unidade básica de saúde em municípios de pequeno porte: instrumentos utilizados, facilidades e dificuldades relacionadas. Gerencia y Políticas de Salud, 2014; 13(27):168-180.

27. SILVA JDF. O enfermeiro no exercício de uma profissão predominantemente feminina: uma revisão integrativa. Monografia (graduação) curso de enfermagem, Universidade Federal do Maranhão, São Luiz, MA. 2018.

28. SILVA VLDS, et al. Práticas de liderança em enfermagem hospitalar: uma self de enfermeiros gestores. Revista da Escola de Enfermagem da USP, 2017; (51) e03206.

29. SOARES MI, et al. Saberes gerenciais do enfermeiro no contexto hospitalar. Revista Brasileira de Enfermagem. 2016; 69(4):631-7.

30. TAVARES SCP. O enfermeiro no exercício de suas competências gerenciais em hospitais públicos da administração direta no município de São Paulo: Análise dos prováveis fatores causadores de vulnerabilidade. São Paulo: Centro Universitário São Camilo, 2010.

31. TORRES E, et al. Sistematização da assistência e gerência do cuidado: Estudo de caso. Escola Anna Nery Revista de Enfermagem 2011; 15(4):730-736.

32. TREVISO P, et al. Competências do enfermeiro na gestão do cuidado. Revista de Administração em Saúde, 2017; 17(69).

33. WILLIG MH, LENARDT MH. A prática gerencial do enfermeiro no processo de cuidar. Cogitare enfermagem, 2002; $7(1): 23-29$.

34. WISNIEWSKI D, et al. Satisfação profissional da equipe de enfermagem x condições e relações de trabalho: estudo relacional. Revista Texto Contexto Enfermagem, 2015; 24(3): 850-8. 